Abstract

\title{
Good and Bad Neighbourhoods in Viral Sequence Space: Predicting, Altering, Targeting Virus Populations ${ }^{\dagger}$
}

\author{
Marco Vignuzzi \\ Viral Populations and Pathogenesis Unit (PVP), CNRS UMR 3569, Institut Pasteur, 28 rue du Dr Roux, \\ 75724 Paris CEDEX 15, France; marco.vignuzzi@pasteur.fr \\ + Presented at Viruses 2020-Novel Concepts in Virology, Barcelona, Spain, 5-7 February 2020. \\ Published: 16 June 2020
}

\begin{abstract}
All viruses, but especially RNA viruses, generate tremendous diversity in genome composition, including point mutations, duplications, deletions, and insertions. We used in vitro and in vivo models to perform natural and directed experimental evolution. We then combined the resulting data with mathematical modelling to determine how virus populations occupy sequence space-a multidimensional hypercube that describes all combinations of nucleotide, codon, or amino acid sequences. In this study, we demonstrate how these experimental and computational approaches can help monitor, predict, alter, and even target virus evolution and population dynamics, creating new ways to study virus-host interactions and to innovate antiviral approaches. Using arboviruses, enteroviruses, and influenza, we recreate and predict host jumps and emergence events in the lab, redirect evolution towards the 'bad' neighbourhoods of sequence space that represent attenuation, and poison the viral population by disturbing the balance between good and bad genomes.
\end{abstract}

Keywords: virus evolution; vaccines; antivirals

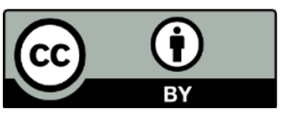

(C) 2020 by the authors. Licensee MDPI, Basel, Switzerland. This article is an open access article distributed under the terms and conditions of the Creative Commons Attribution (CC BY) license (http://creativecommons.org/licenses/by/4.0/). 